\title{
Further studies of reversal learning with singly presented stimuli in pigeons and goldfish*
}

\author{
WILLIAM T. WOODARD and M. E. BITTERMAN \\ University of Hawaii, Honolulu, Hawaii 96822
}

The performance of pigeons and goldfish was studied in a series of discrete-trial red-green discrimination reversals, with stimuli presented singly and the time of response to each measured. Both animals showed progressive improvement, but it took different forms. In the pigeons, there was a decline over reversals in the temporal stability of inhibition and an increase in the rate of inhibitory development. In the goldfish, there was a general increase in speed of response to the positive stimulus. The results are compared with those obtained in previous experiments.

Two recent studies of reversal leaming with singly presented stimuli, one under free operant conditions (Beale, 1970) and the other under discrete-trial conditions (Woodard, Schoel, \& Bitterman, 1971), showed much the same course of improvement in pigeons and much the same pattern of asymptotic performance. One of the changes which occurred as reversal training continued was a decline in the temporal stability of inhibition. At the start of the early reversals, the animals showed considerable reluctance to respond to St (the previously unreinforced stimulus), but there was no such reluctance at the start of the later reversals; that is, the inhibition developed in one session at first carried over to the next session but later tended to decay in the intersession interval. The second major change was an increase in the rate at which inhibition developed within sessions; that is, the rate of decline in readiness to respond to $S-$ was greater in the later than in the earlier reversals. At asymptote, the animals would respond promptly to both stimuli in the early trials of each session and then soon stop responding to $\mathrm{S}-$. The main purpose of the present work with pigeons was to inquire further into the generality of these results. We were interested particularly in whether the dominantly inhibitory character of the changes observed in the previous experiment was due to the fact that reversal training was begun only after strong tendencies to respond to both stimuli had been established by

* This research was supported by Grant MH-17736 from the U.S. Public Health Service, We are indebted to Miss Jennifer Davis for assistance in collecting the data. Requests for reprints should be addressed to the Laboratory of Sensory Sciences. University of Hawaii, 1993 East-West Road. Honolulu, Hawaii 96822 . extensive, consistently reinforced pretraining.

An experiment with goldfish analogous to the discrete-trial experiment with pigeons also showed some progressive improvement, but the course of improvement and the pattern of asymptotic performance in the goldfish both were rather different from those in pigeons (Woodard et al, 1971). The principal change over reversals shown by the goldfish was an overall slowing of response, which may be interpreted as an increase in the general level of inhibition. The improvement in discrimination could be traced primarily to the fact that the slowing was more marked for $S-$ than for $S+$. The animals continued at asymptote to begin each reversal with a considerable reluctance to respond to $\mathrm{S}+$ (the previously unreinforced stimulus), which was overcome gradually by reinforcement, and with a readiness to respond to $S^{--}$(previously reinforced), which was reduced gradually and symmetrically by nonreinforcement. Again, our purpose in the present work was to inquire into the generality of these results.

\section{SUBJECTS}

The Ss were eight male homing pigeons reduced to $80 \%$ of ad lib weight and seven 4 -in. goldfish. The animals-all experimentally naive-were maintained on a $24-\mathrm{h}$ feeding schedule.

\section{APPARATUS}

The apparatus was the same as in the previous experiment (Woodard et al, 1971). The pigeons worked with a panel containing a single Plexiglas key of conventional design, which could be illuminated by colored lamps, and the aperture of a grain magazine. The manipulandum for the goldfish was a Plexiglas key of special design, which could be illuminated with colored lamps and through which licuid food could be pumped. All events of the experiment were programmed automatically, and response times were recorded by a printing counter activated by a pulse generator.

\section{PROCEDURE}

Response to a white key was shaped in discrete trials separated by intervals of darkness. A single contact with the key was reinforced to begin with, and then the response requirement gradually was increased to 20 pecks for the pigeons and 10 presses for the goldfish. When response times had stabilized, the key was illuminated by colored lamps, red on half of each day's 40 trials and green on the remaining trials, in Gellermann orders. Pretraining with the two colors continued for 20 days, but in this experiment (unlike the previous one in which response to both colors was consistently reinforced), response to each color was reinforced on only half the trials, again in Gellermann orders. that is, each color was positive on 10 trials and negative on 10 trials each day.

The procedure for the pigeons on each trial was to illuminate the key with one of the two colors after an intertrial interval of 30 sec in darkness, during which any response to the key was penalized by the resetting of the interval timer. On positive trials, there was no trial limit; the 20th peck (whenever it occurred) turned off the keylight, turned on the white lamp which illuminated the feeder aperture, and presented the grain tray for $3 \mathrm{sec}$, following which the next intertrial interval began. On negative trials, response had no effect; the stimulus stayed on for 30 sec irrespective of the animal's behavior. The measure of performance on each trial was the time in seconds between the presentation of the stimulus and the occurrence of the $20 \mathrm{th}$ peck (if it occurred in $30 \mathrm{sec}$ or less). When fewer than 20 pecks were made in $30 \mathrm{sec}$, a score of $30 \mathrm{sec}$ was recorded. The procedure for the goldfish was the same as for the pigeons, except for the response requirement and the duration of the reinforcement cycle. On a reinforced trial, the 10th contact with the key changed its color to white, caused a drop of food to be delivered, and initiated a 10 -sec period, during which each further contact with the key produced an additional drop of food. For both groups of animals, there were two magazine presentations for purposes of warm-up before the first trial of each session.

In the original problem (Reversal 0), red was consistently reinforced $(S+)$ and green was consistently unreinforced $(S-)$. In the first reversal (Reversal 1), green was $S+$ and red was $\mathrm{S}-$; in Reversal 2, red was $\mathrm{S}+$, and so 


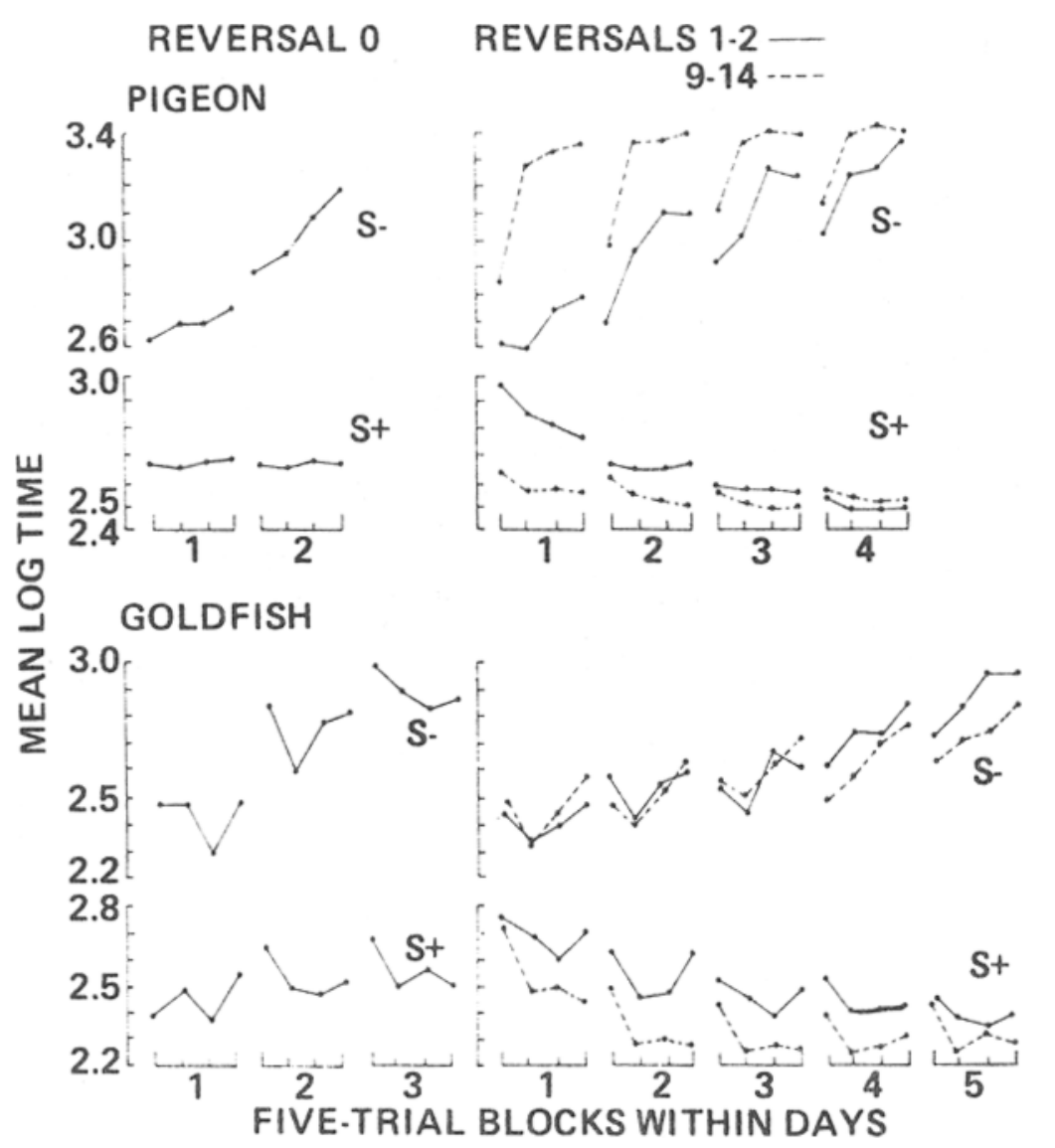

Fig. 1. The discriminative performance of the pigeons and the goldfish at various stages of reversal training.

forth, through Reversal 14. For the pigeons, there were 2 days of training in Reversal 0, but performance was poorer than anticipated on the second day of Reversal 1, and the number of days per reversal was increased to 4. For the goldfish, whose early performance was poorer than that of the pigeons, there were 3 days of training in Reversal 0 and 5 days of training in each of the subsequent reversals.

\section{RESULTS}

The principal results of the experiment are summarized in Fig. 1, which shows the performance of the animals in the original discrimination (Reversal 0), in early reversals (1-2), and at asymptote (Reversals 9-14). The curves are plotted in terms of mean $\log _{e}(t+1)$ per trial for blocks of five trials, where $t=$ time in seconds.

The results for the pigeons are quite like those of our previous experiment (Woodard et al, 1971). The rate of discrimination in Reversal 0 was somerhat less rapid than before (perhaps because of the pretraining with partial reinforcement) and so also was the rate of discrimination in the early reversals (in part perhaps for the same reason and in part because 4 days
A second main factor in the improvement shown by the pigeons was, as before, an increase in the rate of inhibitory development within sessions. The magnitude of this change is masked to some extent by the blocking of trials; the asymptotic rate of inhibitory development found here is even greater, in fact, than that shown in the trial-by-trial plots given in the report of the previous experiment. The significance of the change is shown by an interaction of reversal blocks with trials $(F=3.73$, $\mathrm{df}=18 / 126, p<.01)$ in the data for $\mathrm{S}-$. The same interaction in the data for $\mathrm{S}+$ is negligible.

Again in this experiment, the difference between performance on reversal days (Day 1 ) and nonreversal days (2-5) declined progressively over reversals - there was a highly significant interaction of stimuli with days and reversals $\quad(F=11.26, \quad d f=18 / 126$, $p<.01$-but it did not entirely disappear. At asymptote (Reversals 9-14), there still was significant variation over days in response to $S$ $(F=6.43, \quad d f=9 / 63, \quad p<.01)$, although not in response to $S+$. In this respect, too, the results resemble those obtained before.

The results for goldfish are not, however, like those previously obtained. Progressive improvement appeared once more-the interaction of reversal blocks and stimuli was substantial $(F=4.83, \quad \mathrm{df}=6 / 36$, $p<.01$-but the pattern of change was different. In the previous experiment, improvement over reversals could be traced to a general slowing of response to both stimuli which was greater for S- than for S+. Here, there was a general speeding of response to $S_{+}$. Variation in response time with reversal blocks was highly significant for $\mathrm{S}+\quad(\mathrm{F}=11.54$, df $=6 / 36, p<.01$ ), but not for $S-$ $(F=1.29, \quad \mathrm{df}=6 / 36, \quad p>.05)$. Although the difference between $\mathrm{S}+$ and $\mathbf{S}-$ scores increased over reversals, no clear evidence could be found of a change over reversals in the rate of discrimination within reversals. It should be noted that speed of response to the two stimuli declined substantially in pretraining with the shift from consistent to partial reinforcement, and the animals began Reversal 0 with response speeds much lower than in the previous experiment. There was room here then for increase in speed, which there was not before. DISCUSSION

The present results for pigeons provide further indication of the generality of a pattern of improvement which was shown by pigeons in previous experiments with singly presented stimuli and which has appeared also in experiments with rats 
(Davenport, 1959; Khavari \& Heise, 1967). The improvement in habit reversal shown by these animals in choice situations has two principal components. One is a decline in negative transfer from problem to problem which has been attributed to proactive interference. The results obtained in single-stimulus situations suggest that the change is due to a decline in the stability of inhibition, an idea which is compatible with the interference interpretation on the old Paviovian assumption that inhibition is particularly susceptible to interference. A second principal component of the improvement found in choice situations is an increase in the rate of discrimination within problems. The single-stimulus results suggest that it is due to an increase in the rate of inhibitory development, an idea for which no interpretation of the effect yet offered prepares us. The possibility must, however, be considered that the mechanisms of improvement in the two situations are not entirely the same.

The present results for goldfish are clearly different from those of the previous experiment, perhaps because of the difference in pretraining or the greater amount of training per reversal, but in neither case is the course of improvement or the asymptotic behavior the same as in pigeons. Our own suspicion is that the changes which appear in the two experiments represent nothing more than gradual adjustments in response speed to changing cumulative frequencies of reinforcement and nonreinforcement. In any case, our failure to find in goldfish the pattern of performance which now seems to be characteristic of pigeons suggests that different mechanisms are at work in the two forms.

\section{REFERENCES}

BEAIE I $L$. The effects of amount of training per reversal on successive reversals of a color discrimination. Journal of the Experimental Analysis of Behavior, 1970, 14, 345-352.

DAVENPORT, J. W. Choice behavior and differential response strength in discrimination learning. Journal of Comparative \& Physiological Psy chology. 1959, 52, 349-352.

KHAVARI, K. A., \& HEISE, G. A. Analysis of discrimination reversal in the rat. Psychonomic Science, 1967, 9, 271-272. WOODARD, W. T., SCHOEL, W. M., \& BITTERMAN, M. E. Reversal learning with singly presented stimuli in pigeons and goldfish. Journal of Comparative \& Physiological Psychology, 1971, 76. 460-467. 